\title{
Adaptive control of the filler wire rate during laser beam welding of squared butt joints with varying gap width
}

\author{
Morgan Nilsen $^{1}$ (D) . Fredrik Sikström ${ }^{1} \cdot$ Anna-Karin Christiansson ${ }^{1}$ \\ Received: 30 November 2018 / Accepted: 15 January 2019 / Published online: 21 February 2019 \\ (C) The Author(s) 2019
}

\begin{abstract}
Adding filler wire control to autogenous laser beam welding of squared butt joints offers a means to widen up the tight fit-up tolerances associated with this process. When the gap width varies, the filler wire rate should be controlled to assure a constant geometry of the resulting weld seam. A dual mode sensing system is proposed to estimate the joint gap width and thereby control the filler wire rate. A vision camera integrated into the welding tool together with external LED illumination and a laser line projection enables two sensing modes, one surface feature extraction mode and one laser triangulation-based mode. Data from the both modes are fused in a Kalman filter, and comparisons show that the fusing of the data gives more robust estimation than estimates from each single mode. A feed-forward control system adaptively adjusts the filler wire rate based on the estimations of the joint gap width in front of the keyhole. The focus is on keeping the data processing simple and affordable, and the real-time performance of the sensor and control system has been evaluated by welding experiments. It is shown that the proposed system can be used for on-line control of the filler wire rate to achieve a constant weld geometry during varying joint gap widths.
\end{abstract}

Keywords Laser beam welding $\cdot$ Filler wire $\cdot$ Squared butt joints $\cdot$ Varying gap width $\cdot$ Feature extraction $\cdot$ Laser triangulation . Sensor fusion

\section{Introduction}

Autogenous laser beam welding (LBW) requires tight fit-up tolerances, accurate fixturing, and LBW tool manipulation in order to avoid defects such as lack of fusion, undercut, and underfill. [1]. Even though the joint geometry can be controlled by careful joint preparations, this is costly, so widening the fit-up tolerances can save manufacturing time and money. Adding filler wire to the LBW process makes it less sensitive to variations in joint gap width and hence lowers the demands for the joint tolerances [2]. While autogenous LBW only allows a gap width tolerance of approximately $10 \%$ of the thickness of the work pieces, LBW with filler wire considerably increases the ability to fill the gap. Sun and Kuo [3] showed that a gap width of $1 \mathrm{~mm}$ could be bridged using filler wire when welding 2-mm-thick stainless steel sheets. Yang et al.

Morgan Nilsen

morgan.nilsen@hv.se

1 Department of Engineering Sciences, University West, 46180 Trollhattan, Sweden
[4] performed a study on the correlation between the process parameters (laser power, welding travel speed, and filler wire feed rate) and the weld bead width dimensions. They found that the upper widths of the weld bead and the fusion zone were significantly affected by the filler wire feed rate. However, to achieve a good weld seam geometry and avoid defects when the joint gap width varies, this gap should be measured, and the filler wire feed rate be continuously adapted to the current gap width.

The filler wire can be added either in the leading or trailing welding direction, where the leading direction most often is preferred [5]. However, a leading direction wire feed will obscure the joint in front of the laser interaction zone; hence, optical sensors configured coaxially with the laser beam are not able to measure the joint gap width in that case. Prasad et al. [5] have performed a stability study of an off-axis leading filler wire configuration to enable gap width measurements in front of the laser beam. They showed that this configuration is feasible, which is why it was chosen for the work presented in this paper.

Several researchers have addressed the issue of handling varying joint gap width in LBW. Coste et al. [6] proposed a control system for laser power, travel speed, laser beam 
oscillation, and filler wire rate during $\mathrm{CO}_{2}$ welding of highthickness plates. Measurements of the joint gap width were conducted using a commercial optical sensor from SERVOROBOT, and it was shown that gap widths up to $1.5 \mathrm{~mm}$ could be bridged when welding 8 -mm-thick castings. A model, using a back propagation neural network and a genetic algorithm, was proposed by Zhang et al. [7], describing the relationship between laser power, filler wire feed rate, joint gap width, and misalignment. Experimental results, when measuring the joint gap width using a laser triangulation sensor (distance measurement by angle calculation) during welding, show promising results for obtaining desired geometric properties by using the model together with the gap width measurements. Huang et al. [8] propose an adaptive filler wire feed rate model describing the relationship between welding travel speed, gap width, seam reinforcement, filler wire rate, and laser power. A laser triangulation sensor measures the gap width during welding, and laser power and filler wire rate are controlled according to the proposed model. A laser triangulation-based machine vision system to monitor joint gap width and other features was suggested by Huang and Kovacevic [9]. Position information and geometrical features of the weld joint such as its depth, width, plate misalignment, and cross-sectional area were obtained and monitored in realtime, and a joint tracking implementation was presented. Adaptive filler wire control based on a scanning laserphotoelectric cell sensor and a wire feed controller is suggested by Chen et al. [10]. Welding experiments, where the welding travel speed and filler wire feed rate were controlled, showed that controlling the welding travel speed is preferred with wider gaps while adjusting the filler wire rate is better with narrow gaps.

This paper describes a method using sensor fusion [11] to combine data from a dual mode sensor system to obtain a better result than what would be possible if the sensing modes were used separately. The use of multi-sensor and sensor fusion strategies to monitor and control the LBW process has been presented in several publications, e.g., [12-17], however to the authors' knowledge not for joint gap width estimation and filler wire feed rate control. In this work, a dual mode sensing system, based on a single camera, LED illumination, and a laser line projection, is proposed for estimation of the joint gap width. The system switches between capturing camera images, from either using LED illumination for surface features or from the laser line projection for triangulation. In both cases, the joint gap width is the target.

Measurements from the two sensing modes are fused together to get a more accurate and robust gap width estimate. The filler wire rate is then adapted based on this estimate in order to avoid weld imperfections when the joint gap width varies. Promising results from initial welding experiments using the proposed estimation and control system are presented.

\section{Experimental set-up and procedure}

This chapter describes the set-up of the LBW equipment together with the sensor and control system used in the welding experiments. The material used and the experimental procedure are also described.

\subsection{Laser beam welding set-up}

An industrial robot, ABB IRB4400, was used for LBW tool manipulation. It is a 6-axis robot with a maximum payload of $60 \mathrm{~kg}$, with a position repeatability of $0.19 \mathrm{~mm}$ and a path repeatability of $0.56 \mathrm{~mm}$ (at $1.6 \mathrm{~m} / \mathrm{s})$. The laser source was a 6-kW fiber laser, IPG Ytterbium Fiber Laser (YLR-6000-S) with a wavelength of $1070 \mathrm{~nm}$, and the LBW tool was from Permanova Lasersystem AB. The optical fiber delivery was $600 \mu \mathrm{m}$ in diameter, and the focal length of the collimator and focusing lenses were 160 and $300 \mathrm{~mm}$, respectively. This setup gave a laser beam spot diameter of $1.12 \mathrm{~mm}$ and a Rayleigh length of $13.7 \mathrm{~mm}$. The continuous wave laser power was $2300 \mathrm{~W}$, the welding travel speed was $15 \mathrm{~mm} / \mathrm{s}$, and the laser beam focus was on the surface of the work piece to provide keyhole welding.

A Fronius VR 1500 4R/W/E Roboter was used to feed filler wire in the leading direction, in an off-axis configuration mounted on the robot, see Fig. 1. The filler wire was fed at a $30^{\circ}$ angle in the horizontal plane, and $30^{\circ}$ in relation to the welding direction along the joint. This configuration in the leading direction has been evaluated in detail by Prasad et al. [5]. The reason for using this configuration is that a leading position of the filler wire obscures the top view in front of the joint and makes measurements of the joint gap near the process interaction zone impossible from a camera that is configured coaxial with the processing laser beam. The wire feed rate was calculated to result in a $15 \%$ weld seam reinforcement also with varying gap width as suggested in [5].

Argon was supplied for shielding on the top side of the work piece with a flow rate of $32 \mathrm{l} / \mathrm{min}$ through a $10 \mathrm{~mm}$ in

(a)
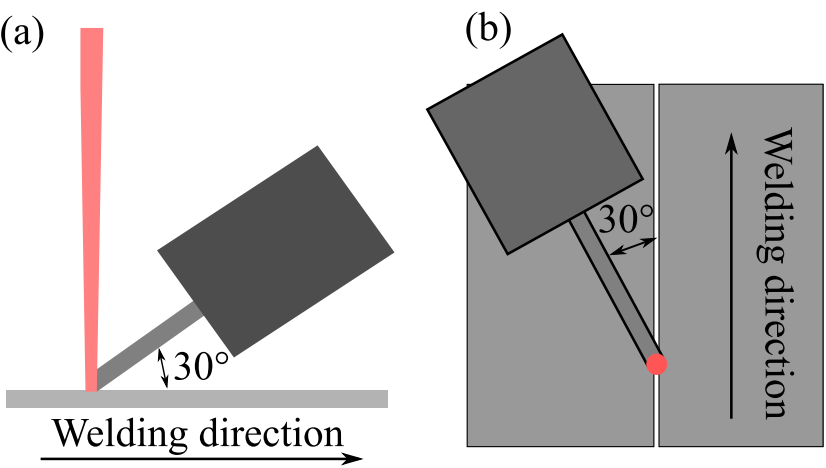

Fig. 1 Filler wire and laser beam configuration. a Side view in the vertical plane in line with the welding direction. b Top view in the horizontal plane coaxial with the laser beam 
diameter tube, and to the root side through a gas channel integrated in the fixture. Argon was also supplied in front of the focusing lens of the LBW tool to protect it against spatter from the process.

\subsection{Sensor and filler wire feeder control}

A dual mode sensor system has been developed utilizing a vision camera, a LED illumination, a laser line projection, and a trigger system for on-line estimation of the gap width. The camera captures top surface features in an area in front of the keyhole, when the scene is illuminated by the LEDs, and a laser line projection, when the scene is illuminated by the laser line. These both modes are activated by the triggering system, which allows the camera to alternate between exposure of the LED illumination and the laser line projection.

The camera was a high dynamic range $(120 \mathrm{~dB}) \mathrm{CMOS}$ camera, coaxially integrated in the LBW tool as shown in Fig. 2. The camera was triggered at a frame rate of 200 frames per second (FPS) (100 FPS for the top surface feature mode and 100 FPS for the laser triangulation mode). The wavelength of the LED illumination and the laser line projection were centered at $450 \mathrm{~nm}$, and a matching optical band pass filter was placed in front of the camera. This wavelength was chosen since the spectral range between 400 to $500 \mathrm{~nm}$ gives minimal disturbances from the process spectral emissions, while still obtaining good sensitivity of the CMOS sensor [18]. This wavelength range is also within the spectral transmittance of the LBW tool optics. The size of the camera image was $640 \times 300 \mathrm{px}$, and the corresponding spatial resolution on

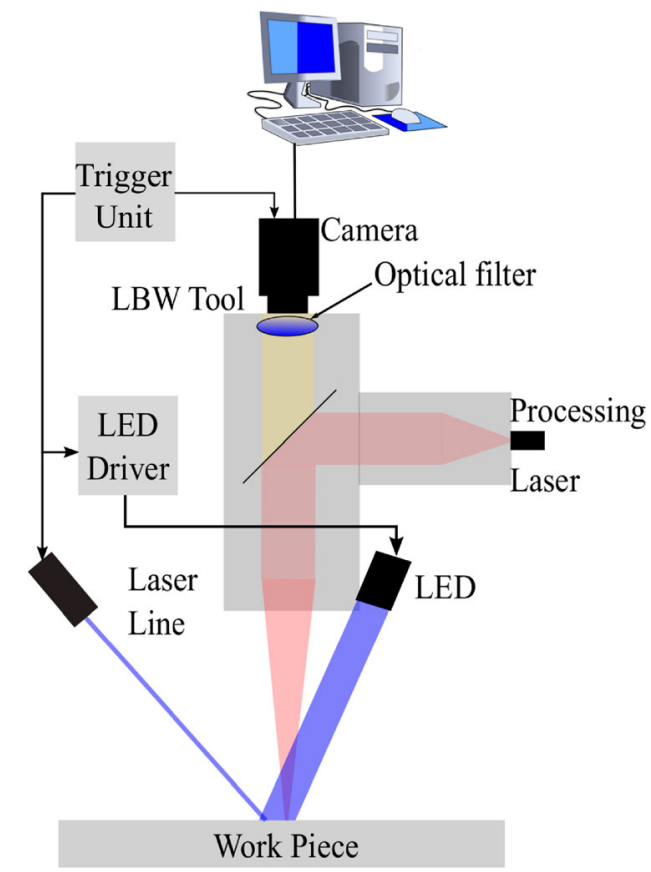

Fig. 2 The LBW tool, trigger unit, camera, and illumination the work piece surface was $20 \mu \mathrm{m} \times 20 \mu \mathrm{m}$ giving a field of view of approximately $13 \mathrm{~mm} \times 6 \mathrm{~mm}$. The LEDs and the laser lines are mounted to move together with the LBW tool by the robot.

The filler wire feeder communicated with the robot controller via a measurement and control computer that could adjust the filler wire rate according to the estimated measurements of the joint gap width.

\subsection{Materials and experimental procedure}

The work piece material was 2-mm-thick sheet metal plates $(40 \mathrm{~mm} \times 300 \mathrm{~mm})$ of stainless steel (SS316). The plates were laser cut to get a squared butt joint configuration with four sections with technical zero gap, and three sections with varying gap width, see Fig. 3 . The sections with varying gap had a maximum gap width of $0.1,0.2$, and $0.3 \mathrm{~mm}$, respectively. The plates were tack welded in the four sections with technical zero gap before welding to avoid distortions during welding. The filler wire was stainless steel (SS316), $1 \mathrm{~mm}$ in diameter, and the wire feed rate was defined by calculations to result in a $15 \%$ weld seam reinforcement as described in [5].

Three different test cases were evaluated:

- Test case 1: Autogenous welding

- Test case 2: Welding with a constant filler wire feed rate

- Test case 3: Welding with an adaptive filler wire feed rate

\section{Gap width estimation}

A data processing algorithm is presented that estimates the gap width by fusing data from the two sensing modes. The first mode captures images of the surface features in front of the keyhole to enable feature extraction. The other mode uses laser triangulation to capture a line profile on the surface perpendicular to the joint, also in front of the keyhole. Data from these two modes are then fused together in a Kalman filter [19] to continuously estimate the joint gap width, here denoted $\hat{\xi}_{c l}$.

\subsection{Image processing of surface feature data}

Figure 4 shows a typical image from the camera while the LED illuminates the scene. Only a small portion of the captured image, a region of interest (ROI) $I_{\mathrm{ROI} c}$ at a short distance in front of the keyhole, is here used to measure the joint gap width $g_{c}$. Limiting $I_{\mathrm{ROI} c}$ to $50 \times 100 \mathrm{px}$ reduces the computational cost, making it suitable for real-time computing.

The gap width $g_{c}$ from the surface feature data in $I_{\text {ROIc }}$ is calculated by first summing the pixel intensity values of each row: 
Fig. 3 Drawing of the work piece used with four segments of technical zero joint gaps and three segments of varying gaps with a maximum of $0.1,0.2$, and $0.3 \mathrm{~mm}$ each

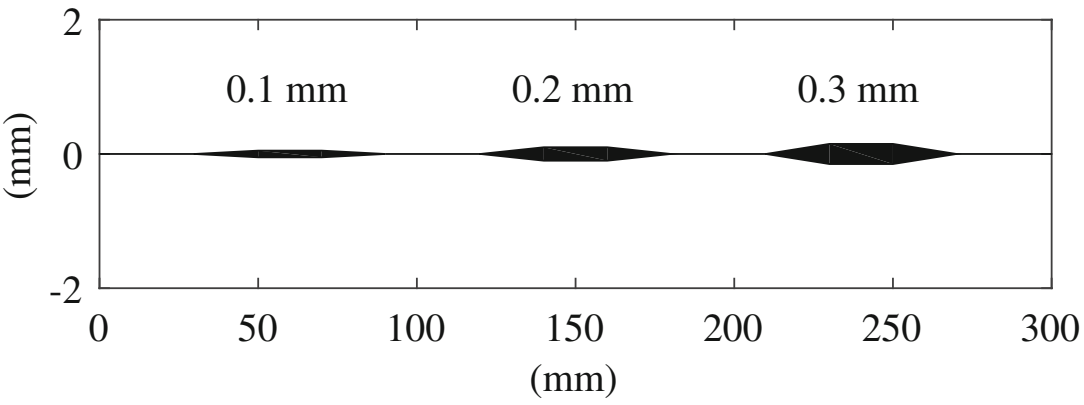

$\alpha_{m}=\frac{1}{N} \sum_{n=1}^{N} I_{\mathrm{ROI} c}\left(y_{m}, x_{n}\right)$

where $\alpha_{m}$ is the intensity sum of row $m$, and $N$ is the number of columns. Applying this to all rows gives an intensity vector $\alpha=\left(a_{m}\right)_{m=1}^{M}$, shown in Fig. 5a, where $M$ is the number of rows in $I_{\mathrm{ROI} c}$. In order to see where possible changes occur, a first derivative of $\alpha$ is calculated as:

$\alpha_{m}^{\prime}=\alpha_{m+1}-\alpha_{m}$

giving a vector of first derivates $\alpha^{\prime}=\left(\alpha_{m}^{\prime}\right)_{m=1}^{M-1}$, see Fig. $5 \mathrm{~b}$. The gap width, $g_{c}$, is defined as the distance between the maximum and minimum derivative, marked by dotted lines in Fig. $5 b$ and c.

The calculation of the derivative vector, $\alpha^{\prime}$, is sensitive to noise in the intensity sum $\alpha$ caused by irregularities in the surface texture and scratches near the joint. A scratch will show up as a large value in $\alpha^{\prime}$, an indication that can be misinterpreted as the joint edge and may lead to false detections. To avoid this, the pixel intensities in $I_{\mathrm{ROI} c}$ are increased by adding an intensity offset based on the mean intensity in every $I_{\text {ROIc }}, I_{\text {mean }}=\frac{1}{M N} \sum_{m=1}^{M} \sum_{n=1}^{N} I_{\text {ROI } c}\left(y_{m}, x_{n}\right)$ making the surface area apart from of the joint area in every acquisition saturated. The result is shown in Fig. 6. This measure will effectively remove the noise from the image and improve the robustness of the estimates.
To avoid false detections in segments where tack welds obscures the joint, automatic detection of the presence of a tack weld is implemented. The mean intensity $I_{\text {mean }}$ is calculated in the current $I_{\mathrm{ROI} c}$ and then compared with the $I_{\text {mean }}$ from the previous $I_{\mathrm{ROI} c}$. If the change in mean intensity between two consecutive images is larger than a threshold, $T_{i}$, it is assumed that a tack weld (or some other disturbance) is present and the gap width measurement $g_{c}$ is not trusted. The estimated variance of $g_{c}, R_{c}$, is used to convey this information to the Kalman filter:

$R_{c} \in \begin{cases}\frac{1}{\mathrm{I}} \sum_{\iota=1}^{\mathrm{I}}\left(r_{\iota}-\bar{r}\right)^{2} ; & I_{\text {mean }, k}-I_{\text {mean }, k-1} \leq T_{t} \\ 10^{6} ; & I_{\text {mean }, k}-I_{\text {mean }, k-1}>T_{t}\end{cases}$

where $r_{\iota}=g_{r, \iota}-g_{c, \iota} \in \mathrm{O}$, a set of in total $I$ residuals between estimated gap width measurements $g_{c, \iota}$ and a reference $g_{r, \iota}$, and $\bar{r}=\frac{1}{\mathrm{I}} \sum_{\iota=1}^{\mathrm{I}} r_{\iota}$. The reference gap width $g_{r, \iota}$ in $\mathrm{O}$ has been acquired by $I$ manual measurements of the gap width in a sequence of images with no tack welds.

\subsection{Image processing of the laser triangulation data}

Figure 7 shows a typical image from the camera while the laser line illumination is on, and it is used for laser triangulation to calculate a line profile on the surface across the joint. A ROI is selected, $I_{\mathrm{ROI}}$, around the intersection point of the
Fig. 4 Image with the LED illuminated scene in the camera coordinate system

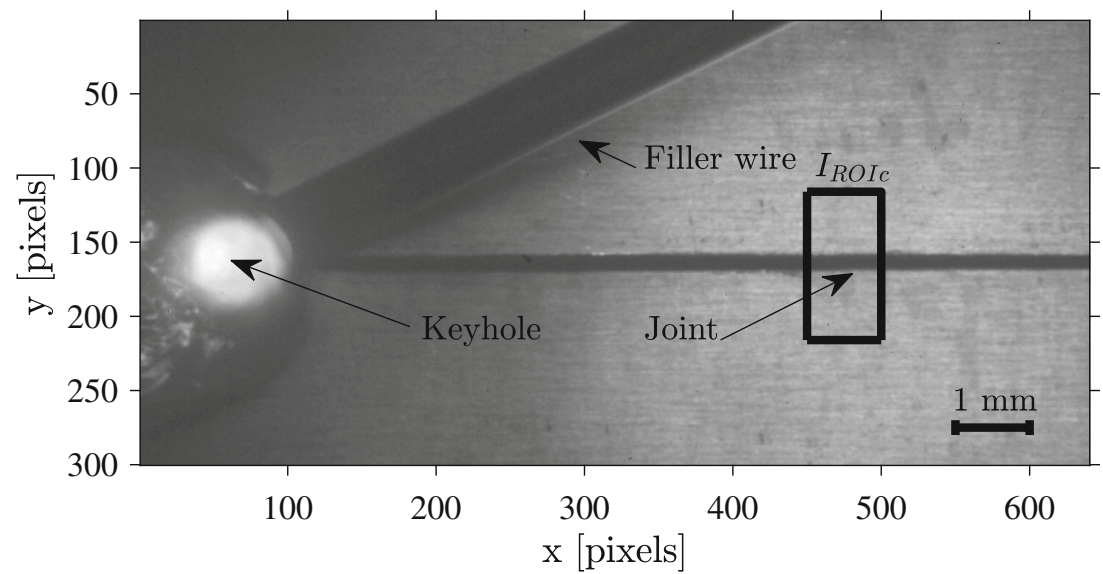


(a)

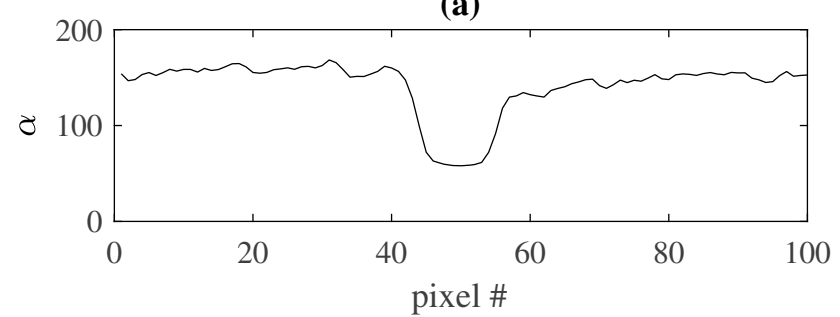

(b)

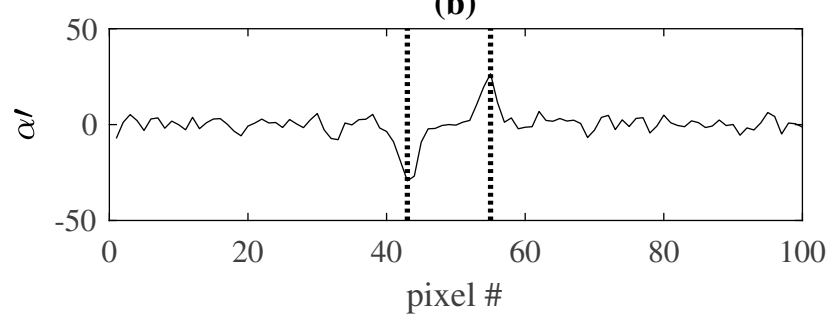

(c)

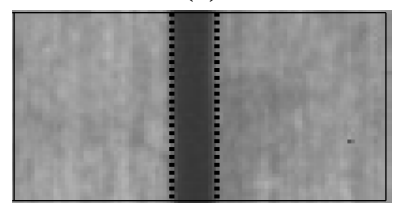

Fig. 5 a The intensity vector $\alpha$. b The first derivative of $\alpha, \alpha$, where the estimated gap width is marked by the vertical dotted lines. c The image $I_{\text {ROI }}$, rotated $90^{\circ}$, where the gap width is indicated by the vertical dotted lines

(a)



(b)

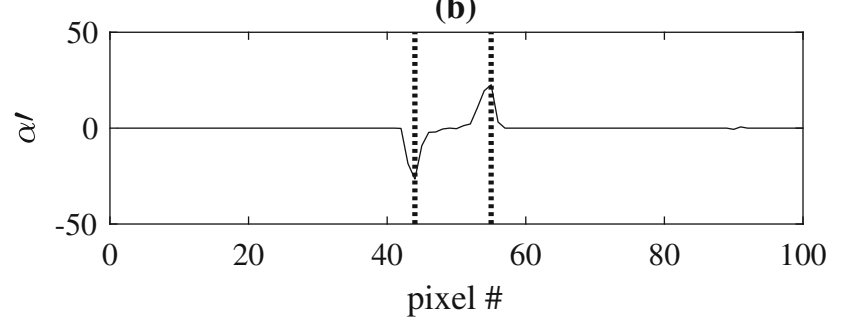

(c)



Fig. 6 The pixel intensity of $I_{\text {ROIc }}$, has been increased. a The pixel intensity profile, $\alpha . \mathbf{b} \alpha^{\prime}$, where the max and min value of $\alpha^{\prime}$ is marked by the vertical dotted lines. c The image $I_{\text {ROIc }}$, rotated $90^{\circ}$, where the gap width is indicated by the vertical dotted lines projected laser line and the joint gap, and it is used to calculate the gap width measurement $g_{l}$.

$I_{\mathrm{ROI} /}$ is binarized into a black and white image, and the pixels corresponding to the laser line are then thinned to have a width of just $1 \mathrm{px}$ to obtain a line profile. Thinning is conducted by selecting the first and the last white pixel for each row in $I_{\mathrm{ROI} l}$ and then selecting the median to represent the line profile. However, if the width of the white laser line is below a threshold, $T_{l}$, this row data is associated to the joint gap. In this fashion, the gap width, $g_{l}$, is given by the number of rows that is associated with the gap. If the triangulation measurement is disturbed, e.g., by a tack weld, the total intensity (sum of all the pixel intensity values in $I_{\mathrm{ROI}}$ ) will considerably change. Hence, a threshold value, $T_{d}$, is used, and when the average intensity of $I_{\mathrm{ROI} l}$ is below this value, it is assumed that a tack weld (or some other disturbance) is present, and the gap width measurement $g_{l}$ is not trusted. The measurement of $g_{l}$ and its corresponding variance $R_{l}$ are then treated in a similar fashion as the measurement of $g_{c}$ in the Kalman filter.

\subsection{Sensor fusion implementation}

The gap width measurements from the two sensing modes, i.e., $g_{c}$ and $g_{l}$, are fused together in an implementation of a Kalman filter where the gap width measurements $g_{c}$ and $g_{l}$ together with the respective variances, $R_{c}$ and $R_{l}$ are used as inputs. The model used in the filter assumes that the gap width is constant between two consecutive image frames; however, a noise component is added to allow for a certain change. The state transition model is given by:

$\xi_{k+1}=\xi_{k}+T w_{k}$

where the scalar state vector $\xi_{k}$ represents the joint gap width at time instant (image frame index) $k$ and $w_{k} \in N(0$, $Q$ ) is the state noise, and $Q$ is the state noise variance. $T$ is the time between two image frames. In the case of one single measurement, the observation equation is:

$\hat{g}_{k}=\xi_{k}+R_{k} v_{k}$

where $\hat{g}_{k}$ is the measured gap width at time instant $k$, $R_{k} \in\left\{R_{c, k}, R_{l, k}\right\}$, is the weight of the respective variances, and $v_{k} \in N(0,1)$ is the measurement noise for each mode and $C=1$.

When using two sensing modes in the filter, the observation equation is:

$\hat{g}_{k}=C \xi_{k}+R_{k} v_{k}=\left[\begin{array}{l}1 \\ 1\end{array}\right] \xi_{k}+\left[\begin{array}{cc}R_{c, k} & 0 \\ 0 & R_{l, k}\end{array}\right] v_{k}$ 
Fig. 7 Image when the laser line illumination is active. $I_{\mathrm{ROI} l}$ is marked by the black rectangle in the camera coordinate system

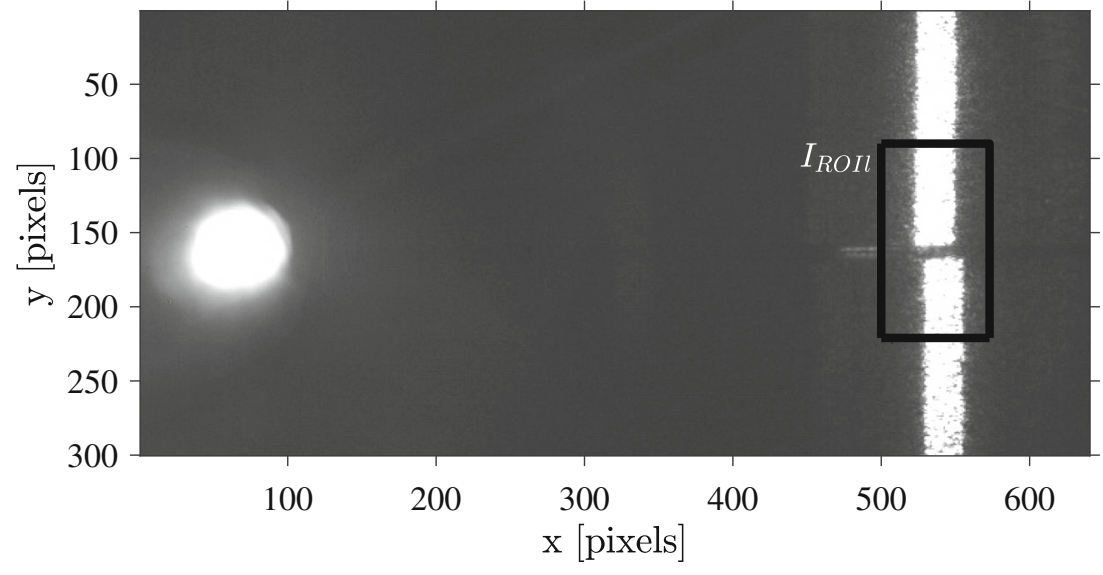

The Kalman filter is defined by a measurement update Eq. (7) and a time update (prediction) Eq. (8)

$$
\begin{gathered}
\hat{\xi}_{k \mid k}=\hat{\xi}_{k \mid k-1}+P_{k \mid k-1} C^{T}\left(C P_{k \mid k-1} C^{T}+R_{k}\right)^{-1}\left(g_{k}-C \hat{\xi}_{k \mid k-1}\right) \\
P_{k \mid k}=P_{k \mid k-1}-P_{k \mid k-1} C^{T}\left(C P_{k \mid k-1} C^{T}+R_{k}\right)^{-1} C P_{k \mid k-1} \\
\hat{\xi}_{k+1 \mid k}=\hat{\xi}_{k \mid k} \\
P_{k+1 \mid k}=P_{k \mid k}+T^{2} Q
\end{gathered}
$$

where $\hat{\xi}_{k}$ is the mean value, and $P_{k}$ the covariance of the new normally distributed random variable based on the measured gap widths $g_{k} \in\left\{g_{c, k}, g_{l, k}, g_{c l, k}\right\}$ where $g_{c l k}$ is defined as:

$$
\begin{aligned}
& g_{c l 1}=\left[\begin{array}{c}
g_{c 1} \\
0
\end{array}\right], g_{c l 2}=\left[\begin{array}{l}
g_{c 1} \\
g_{l 2}
\end{array}\right], g_{c l 3}=\left[\begin{array}{l}
g_{c 3} \\
g_{l 2}
\end{array}\right], \\
& g_{c l 4}=\left[\begin{array}{l}
g_{c 3} \\
g_{l 4}
\end{array}\right], g_{c l 5}=\left[\begin{array}{l}
g_{c 5} \\
g_{l 4}
\end{array}\right], \ldots
\end{aligned}
$$

i.e., a new update of data from each mode is used with the previous data from the other mode as the data is updated with a new camera image.

The output from the system is the estimated gap width $\hat{\xi}_{k}$ in front of the keyhole, which is used in next section to adjust the filler wire feed rate to an appropriate value.

\subsection{Adaptive control of filler wire feed rate}

Variations in joint gap width are measurable perturbations from the nominal welding situation with a constant gap width. The filler wire feed rate is led by feed-forward of the estimated gap width as shown in Fig. 8. The nominal filler wire feed rate, $v$, is thus adjusted by $\Delta v$, based on the estimated gap width, $\hat{\xi}_{k}$. The control scheme includes the transfer function $G_{1}(s)$ representing the dynamics of the sensing arrangement and $G_{2}(s)$ representing the wire feed mechanism. The transfer function $G_{1}(s)=e^{\tau_{d 1} s}$ models a time shift, a consequence of the arrangement where the gap width estimation is captured in $I_{\mathrm{ROI} l}$ and $I_{\mathrm{ROI}}$, at a distance $d$ ahead of the keyhole. The time $\tau_{d 1}$ is consequently a function of the welding travel speed $\nu_{t}$ and the distance $d$, such as $\tau_{d 1}=d / \nu_{t}$. The transfer function $G_{2}$ $(s)=\frac{e^{-\tau} \tau 2 s}{1+\tau s}$ models the delay time and a time constant in the wire feed mechanism $\left(\tau_{d 2}=0.01, \tau=0.144\right)$. This transfer function has been found by experimental system identification [20]. When $\tau_{d 2}$ is known and the welding travel speed $\nu_{t}$ is decided, this information should in turn decide the distance $d$ such that $\tau_{d 2}-\tau_{d 1}=0$.

The adaptive filler rate control enables a more consistent weld seam geometry compared to welding with a constant filler rate when the joint gap width varies along the joint.

\section{Results}

The sensing system and adaptive control law have been tested during several welding experiments. Table 1 gives

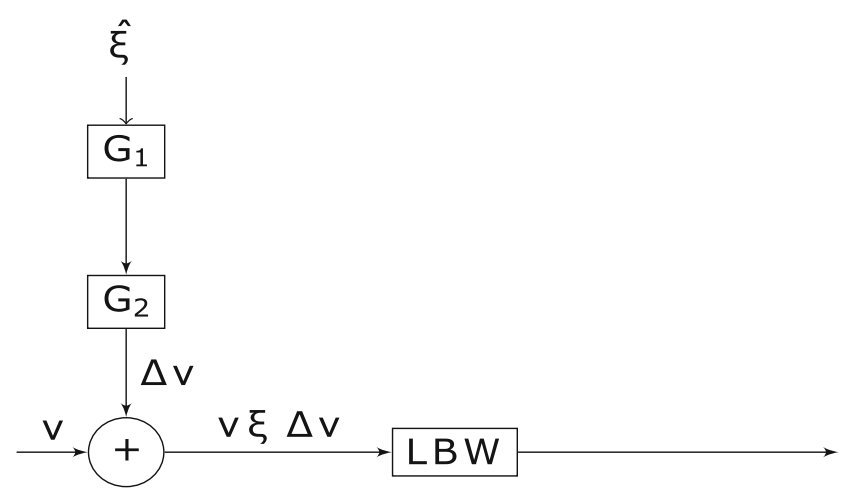

Fig. 8 Feed-forward control of the filler wire rate $v$ 
Table 1 Parameters used during welding experiments

\begin{tabular}{cccccc}
\hline$T_{i}$ & $R_{c}$ & $T_{l}$ & $R_{l}$ & $T_{d}$ & $Q$ \\
\hline 1 & 1 & 20 & 1 & 120 & 0.01 \\
\hline
\end{tabular}

the parameter values used in the data processing algorithm described in Sect. 3. The threshold for change in intensity between two consecutive image frames, $T_{i}$, has been experimentally derived to obtain a sufficient estimation performance. The threshold for the triangulation laser line width, $T_{l}$, has been defined by measuring the nominal width of the laser line in the captured images. The threshold for the average intensity of $I_{\mathrm{ROI}}, T_{d}$, has been defined by comparing the average intensity when only the joint gap is present with the case where a tack weld is visible in $I_{\mathrm{ROI}}$. The state noise variance $Q$ has been experimentally derived to obtain a sufficient estimation performance.

\subsection{Gap width estimation}

Gap width estimations for the three different test cases defined in Sect. 2.3 are presented, and comparisons are made of estimates using a single sensing mode, either the surface feature data, $\hat{\xi}_{c}$, or the laser triangulation data, $\hat{\xi}_{l}$, and also in a combination of both sensing modes, $\hat{\xi}_{c l}$. Figure 9 shows the raw, unfiltered measurement from surface feature extraction, $g_{c}$, and the laser triangulation sensing, $g_{l}$, from a complete welding of test case 1 (welding without filler wire control). The measurements are noisy, and large disturbances in the laser triangulation measurements, $g_{l}$, is visible in the four joint positions of the tack welds. The filtered laser triangulation measurements, $\hat{\xi}_{l}$, surface feature estimate, $\hat{\xi}_{c}$, and the fused estimate from both sensing modes, $\hat{\xi}_{c l}$, are shown in Fig. 10 together with the reference measurement, $g_{r}$. The

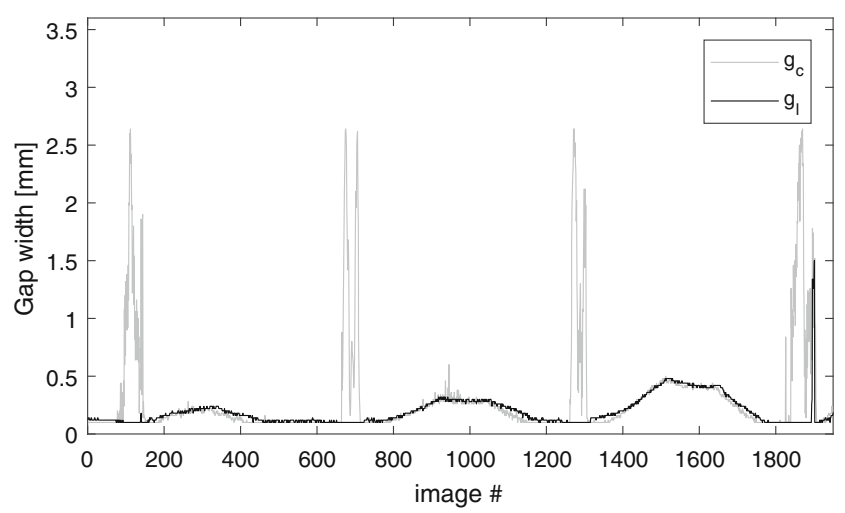

Fig. 9 Unfiltered data from the surface feature extraction $g_{c}$ and the laser triangulation $g_{l}$ during a complete welding sequence measurements are less noisy, and the outliers caused by tack welds are eliminated. The laser triangulation sensing mode, $\hat{\xi}_{l}$, slightly underestimates the gap width while the surface feature measurements, $\hat{\xi}_{c}$, overestimates. By fusing the two sensing modes together, a better fit between the estimate, $\hat{\xi}_{c l}$, and reference, $g_{r}$, is obtained.

A summary of the test cases is given in Table 2 where the maximum and mean error, the standard deviation, and the 1-norm of the error are presented. The errors in the laser triangulation estimate, $\hat{\xi}_{l}$, and the surface feature estimate, $\hat{\xi}_{c}$, are similar in test case 1 , although the surface feature estimate, $\hat{\xi}_{c}$, shows a slightly better fit. The results from the fused estimation, $\hat{\xi}_{c l}$, are however better in all comparisons. The maximum error in the surface feature sensing, $\hat{\xi}_{c}$, is larger than in the laser triangulation estimate, $\hat{\xi}_{l}$, in test case 2 . The mean error is the same, and the standard deviation and the 1-norm are comparable. The maximum error of the fused estimate, $\hat{\xi}_{c l}$, is larger than the error in laser triangulation estimate but smaller than the error in the surface feature estimate, and the fused data estimate shows better results in all other cases. The laser triangulation estimates are better than the surface feature estimate in test case 3 . The laser triangulation estimate gives the smallest maximum error in this case, but the fused data estimate gives the best results in all other cases.

Figure 11 shows histograms of the total sum of errors during all experimental evaluations. Observations of Fig. 10 indicated that the laser triangulation slightly underestimates the gap width while the surface feature extraction sensing is somewhat overestimated. This is confirmed by the histogram in Fig. 11, and it also shows that the distribution of errors in the fused data case is better centered around zero.



Fig. 10 Data from the surface feature sensing mode, $\hat{\xi}_{c}$, laser triangulation sensing mode, $\hat{\xi}_{l}$, fused data $\hat{\xi}_{c l}$, and the reference measurements, $g_{r}$, for a complete welding sequence 
Table 2 Summary of the results from the three different test cases

\begin{tabular}{lllll}
\hline $\begin{array}{l}\text { Test case no./ } \\
\text { sensing mode }\end{array}$ & $\begin{array}{l}\text { Max } \\
\text { error }\end{array}$ & $\begin{array}{l}\text { Mean } \\
\text { error }\end{array}$ & $\begin{array}{l}\text { Standard } \\
\text { deviation }\end{array}$ & 1-norm \\
\hline 1/Laser triangulation & 0.078 & 0.014 & 0.020 & 27.5 \\
1/Surface feature & 0.075 & 0.013 & 0.019 & 25.6 \\
1/Fused data & 0.057 & 0.010 & 0.016 & 20.3 \\
2/Laser triangulation & 0.030 & 0.010 & 0.013 & 18.3 \\
2/Surface feature & 0.056 & 0.010 & 0.016 & 18.3 \\
2/Fused data & 0.040 & 0.007 & 0.012 & 14.4 \\
3/Laser triangulation & 0.019 & 0.010 & 0.013 & 20.5 \\
3/Surface feature & 0.121 & 0.016 & 0.020 & 30.6 \\
3/Fused data & 0.036 & 0.008 & 0.011 & 15.1 \\
\hline
\end{tabular}

\subsection{Adaptive wire feed rate control}

The work piece weld seam geometry has been scanned on the top surface using a 3D-scanning system (ATOS Core 80 ) to evaluate the performance of the wire feed rate control. Figure 12 shows typical results from the scanned surfaces of two of the welded work pieces at the position where the joint gap width was $0.3 \mathrm{~mm}$. The result from

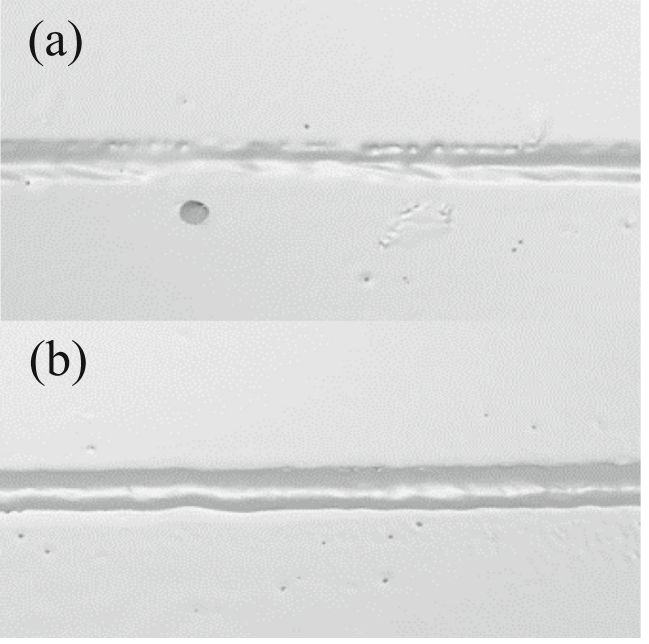

Fig. 12 3D-scanning of the top surface of the weld seam when the gap width was $0.3 \mathrm{~mm}$. a Constant filler wire rate. b Adaptive filler wire rate

using a constant filler wire rate (test case 2) is shown in Fig. 12a, and the result from using adaptive filler wire rate is shown in Fig. 12b. It is clear that the geometry of the weld seam (weld reinforcement) is significantly more consistent when using an adaptive filler wire rate. However, a constant filler wire rate results in a weld seam geometry
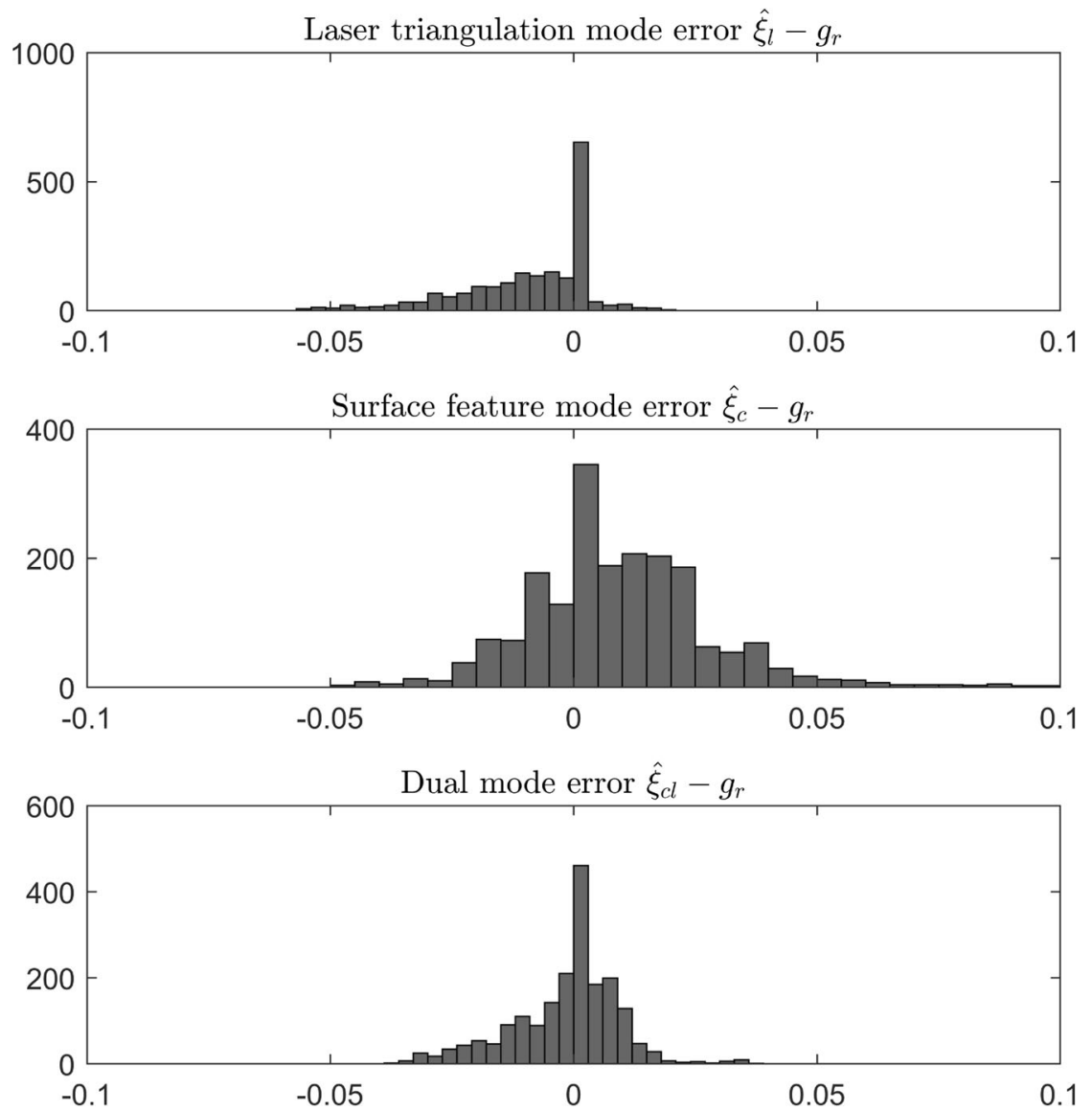

Fig. 11 The distribution of errors, in mm, in all gap width estimations in the three sensing modes $\left(\hat{\xi}_{c}, \hat{\xi}_{l}, \hat{\xi}_{c l}\right)$ 




Fig. 13 Weld seam reinforcements along the 280 -mm weld

that is significantly affected by joint gap changes when going from a closed gap to a $0.3-\mathrm{mm}$ gap width

A laser triangulation scanner (scanCONTROL LLT281025) has been used to measure the seam reinforcement along the entire weld seam, and the results are shown in Fig. 13. The reinforcement is almost constant along the first $100 \mathrm{~mm}$ of the joint path (before the position when the 0.2-mm gap width starts). The reinforcement is decreased when welding with a constant filler wire rate (test case 2) along the 0.2-mm gap width segment, approximately between the joint positions 100 and $150 \mathrm{~mm}$. The opposite is true when using an adaptive filler wire rate (test case 3 ), then a significant increase of the reinforcement is visible. In the $0.3-\mathrm{mm}$ gap width segment, approximately between the joint positions 200 and $250 \mathrm{~mm}$, the difference in reinforcement between both these cases is even more prominent.

\section{Discussion}

Using an optical sensor system integrated in the LBW tool to measure the joint gap width during LBW is challenging due to significant process disturbances. High intensity spectral emissions from the process can easily saturate the detectors, and evaporated material and smoke from the keyhole may obscure the field of view. Besides, there could typically be surface scratches on the work piece near the joint that would interfere the measurements, and tack welds that completely would obscure the joint. These issues have been addressed in this work, and a sensor system and a data processing algorithm are relatively insensitive to those disturbances is presented in Sect. 3 .

The results from the welding experiments show that both the surface feature mode and the laser triangulation mode are able to measure the joint gap width with a mean error less than $0.016 \mathrm{~mm}$. Although the two sensor modes show good accuracy used separately, fusing the measurements significantly improves the estimation performance, with a mean error less than $0.01 \mathrm{~mm}$.
Results from measurements of the weld seam reinforcement, when the filler wire rate was constant, showed that the reinforcement was decreased as the gap became wider. This was expected since at wider gaps, the volume to be filled increases, and with constant filler wire rate, the gap cannot be successfully bridged. However, with controlled filler wire rate, the reinforcement was increased when the gap width increased. This indicates that too much filler wire was added to compensate for the gap width, and that the system needs to be tuned. Besides form tuning of the filler wire rate, the system showed promising results for avoiding defects when welding squared butt joint with varying gap width.

\section{Conclusions and future work}

Using high power LED and laser line projection at a wavelength of $450 \mathrm{~nm}$, where a reduced amount of spectral process emissions is present, a matching optical bandpass filter in front of the camera, and a camera with high dynamic range made it possible to obtain good image information of the area in front of the melt pool where the joint is located. Increasing the intensity of the gray scale image from the camera, so that the area outside the joint becomes saturated, made it less sensitive to scratches enabling more robust measurements of the gap width. The issue of tack welds was addressed by analyzing the average intensity in the camera images. Normally, the mean intensity of two consecutive images should not change very much; however, when something disturbs the measurement, e.g., a tack weld, the change in mean intensity of the image is larger. This enabled detection of tack welds, and the estimated gap width could still be robust by assuming that the gap width does not change during welding over the tack weld.

A Kalman filter used to enable model-based estimation and sensor fusion of the dual mode sensing proved that sensor fusion gives more accurate estimates than single mode sensing.

Adaptive filler wire control resulted in a more consistent weld seam geometry even in the presence of gap width variations.

Future work includes more welding experiments with larger gap widths to further verify the robustness of the system. Tuning of the system should also be conducted in order to obtain a constant reinforcement of the weld seam, independently of the variations in joint gap width.

Acknowledgments This work was supported by the VINNOVA project VarGa (2016-03291).

Open Access This article is distributed under the terms of the Creative Commons Attribution 4.0 International License (http:// creativecommons.org/licenses/by/4.0/), which permits unrestricted use, distribution, and reproduction in any medium, provided you give 
appropriate credit to the original author(s) and the source, provide a link to the Creative Commons license, and indicate if changes were made.

Publisher's note Springer Nature remains neutral with regard to jurisdictional claims in published maps and institutional affiliations.

\section{References}

1. Steen WM, Mazumder J (2010) Laser material processing. Springer London, London

2. Dilthey U, Fuest D, Scheller W (1995) Laser welding with filler wire. Opt Quant Electron 27(12):1181-1191

3. Sun Z, Kuo M (1999) Bridging the joint gap with wire feed laser welding. J Mater Process Technol 87(1):213-222

4. Yang D, Li X, He D, Nie Z, Huang H (2012) Optimization of weld bead geometry in laser welding with filler wire process using Taguchi's approach. Opt Laser Technol 44(7):2020-2025

5. Siva Prasad H, Frostevarg J, Kaplan AFH (2019) The stability of laser welding with an off-axis wire feed. J Mater Process Technol 264:84-90

6. Coste F, Fabbro R, Sabatier L (1999) Adaptive control of highthickness laser welding. Weld Int 13(6):465-469

7. Zhang K, Chen Y, Zheng J, Huang J, Tang X (2017) Adaptive filling modeling of butt joints using genetic algorithm and neural network for laser welding with filler wire. J Manuf Process 30:553-561

8. Huang J, Zhang K, Zhu X, Tang X (2016) Robot-based adaptive laser wire welding of ship steel plates. Presented at 2016 IEEE Workshop on Advanced Robotics and its Social Impacts (ARSO), pp 170-173

9. Huang W, Kovacevic R (2012) Development of a real-time laserbased machine vision system to monitor and control welding processes. Int J Adv Manuf Technol 63(1-4):235-248
10. Chen W, Liu C, Yang W, Zhang X (2002) Gap-detecting sensor and wire feed control in butt laser welding with filler wire. Lasers Mater Process Manuf 4915:208-218

11. Gustafsson F (2015) Statistical sensor fusion. Studentlitteratur, Lund

12. You D, Gao X, Katayama S (2014) Multisensor fusion system for monitoring high-power disk laser welding using support vector machine. IEEE Trans Ind Inform 10(2):1285-1295

13. Zhang P, Kong L, Liu W, Chen J, Zhou K (2008) Real-time monitoring of laser welding based on multiple sensors. Presented at 2008 Chinese Control and Decision Conference, 2008, pp 1746-1748

14. Al-Habaibeh A, Shi F, Brown N, Kerr D, Jackson M, Parkin RM (2004) A novel approach for quality control system using sensor fusion of infrared and visual image processing for laser sealing of food containers. Meas Sci Technol 15(10):1995-2000

15. Zeng J, Chang B, du D, Wang L, Chang S, Peng G, Wang W (2018) A weld position recognition method based on directional and structured light information fusion in multi-layer/multi-pass welding. Sensors 18(1):129

16. Sun A, E. K.-A. Jr, Gartner M (2002) Monitoring of laser weld penetration using sensor fusion. J Laser Appl 14(2):114-121

17. Gao X, Sun Y, You D, Xiao Z, Chen X (2016) Multi-sensor information fusion for monitoring disk laser welding. Int J Adv Manuf Technol 85(5):1167-1175

18. Sikström F, Nilsen M, Eriksson I (2014) Optical methods for inprocess monitoring of laser beam welding. Presented at the 6th Swedish Production Symposium, pp 1-9

19. Kalman RE (1960) A new approach to linear filtering and prediction problems. J Basic Eng 82(1):35-45

20. Ljung L (1999) System identification: theory for the user, 2nd edn. Prentice Hall, Upper Saddle River 